QUARTERLY OF APPLIED MATHEMATICS

VOLUME LXVII, NUMBER 1

MARCH 2009, PAGES 93-111

S 0033-569X(09)01145-X

Article electronically published on January 7, 2009

\title{
EXPONENTIAL AND POLYNOMIAL DECAY FOR FIRST ORDER LINEAR VOLTERRA EVOLUTION EQUATIONS
}

\author{
BY
}

EDOARDO MAININI (Classe di Scienze Scuola Normale Superiore Piazza dei Cavalieri 7, I-56126

Pisa, Italy)

AND

GIANLUCA MOLA (Dipartimento di Matematica "F.Brioschi" Politecnico di Milano Via Bonardi 9, I-20133 Milano, Italy \& Department of Applied Physics, Graduate School of Engineering, Osaka University, Suita, Osaka 565-0871, Japan)

Abstract. We consider, in an abstract setting, an instance of the Coleman-Gurtin model for heat conduction with memory, that is, the Volterra integro-differential equation

$$
\partial_{t} u(t)-\beta \Delta u(t)-\int_{0}^{t} k(s) \Delta u(t-s) d s=0 .
$$

We establish new results for the exponential and polynomial decay of solutions, by means of conditions on the convolution kernel which are weaker than the classical differential inequalities.

1. Introduction. Given a real Hilbert space $(H,\langle\cdot, \cdot\rangle,\|\cdot\|)$, we consider the following linear homogeneous Volterra integro-differential equation of the first order:

$$
\left\{\begin{array}{l}
\partial_{t} u(t)+\beta A u(t)+\int_{0}^{t} k(s) A u(t-s) d s=0, \quad t>0 \\
u(0)=u_{0} \in H
\end{array}\right.
$$

Here $\beta>0$ and $A$ is a strictly positive selfadjoint operator on $H$ with domain $\mathcal{D}(A)$. The memory kernel $k$ is a piecewise smooth convex decreasing function on $\mathbb{R}^{+}=(0, \infty)$, summable along with its (distributional) derivative.

In the concrete formulation,

$$
H=L^{2}(\Omega), \quad A=-\Delta, \quad \mathcal{D}(A)=H_{0}^{1}(\Omega) \cap H^{2}(\Omega),
$$

Received July 9, 2007.

2000 Mathematics Subject Classification. Primary 35B41, 37L30, 45J05, 80A22.

The second author was supported by the Postdoctoral Fellowship of the Japan Society for the Promotion of Sciences (No. PE06067).

E-mail address: edoardo.mainini@sns.it

E-mail address: gianluca.mola@polimi.it 
equation (1.1) describes the evolution of heat flow in an isotropic rigid heat conductor occupying a bounded smooth volume $\Omega \subset \mathbb{R}^{3}$. The unknown function $u$ is the absolute temperature in the body, according to the so-called Coleman-Gurtin conduction law [5].

Problem (1.1) has been studied by many authors, both for the sake of well-posedness and stability issues, and it is well known (see, e.g., [3, 8, 10, 12, 14]) that, for every $u_{0} \in H$, it possesses a unique weak solution $u \in C([0, \infty), H)$.

Energy dissipation. The main task of this paper is to establish, under different requirements on the memory kernel $k$, uniform decay properties of the system energy, defined by

$$
\mathcal{E}(t)=\|u(t)\|^{2}-\int_{0}^{\infty} k^{\prime}(s)\left\|\int_{t-s}^{t} u(y) d y\right\|_{V}^{2} d s,
$$

having set $u(t)=0$ for $t<0, V=\mathcal{D}\left(A^{1 / 2}\right)$ and $\|\cdot\|_{V}=\left\|A^{1 / 2} \cdot\right\|$. As we will show later, this is the actual form for the energy, where the integral term accounts for past values of the variable $u$. The dissipativity of (1.1) lies in the fact that $\mathcal{E}(t)$ is a decreasing function. To be more precise, we introduce the following

Definition 1.1. Let $\Lambda:[0, \infty) \rightarrow[0, \infty)$ be a decreasing vanishing function. We say that $\mathcal{E}(t)$ has a (uniform) decay rate $\Lambda$ if there exists an increasing positive $\mathcal{Q}$ such that

$$
\mathcal{E}(t) \leq \mathcal{Q}(\mathcal{E}(0)) \Lambda(t) .
$$

In particular, we shall investigate the following instances:

- $\Lambda(t)=e^{-\varepsilon t}$ for some $\varepsilon>0$ [exponential stability],

- $\Lambda(t)=(1+t)^{-p}$ for some $p>0$ [polynomial stability of rate $p$ ].

From now on, the stability of problem (1.1) is understood to correspond to the one of $\mathcal{E}$, according to the above definition. Moreover, $\mathcal{Q}$ will always denote a generic positive constant, possibly varying within the same formula, and (increasingly) depending only on $\mathcal{E}(0)$.

Energy decay results have already been achieved in linear viscoelasticity with memory, both for the infinite delay [18] and for the Volterra [6] equation, under very general assumptions on the memory kernel. We point out that investigations therein exploited rely on the so-called past history setting. Such an approach, originally raised in [7, is necessary in order to treat the infinite delay case in the framework of the semigroup of operators, on a suitable phase-space. Moreover, this tool seems to be very useful even for a Volterra-type equation, where the semigroup generation cannot be expected (and then non-exponential uniform decay is possible). In either problems [18] and [6], without requiring the kernel to fulfill any differential inequality, exponential stability is achieved, as well as polynomial stability in the Volterra case. We stress that in both the above quoted examples, such results can be established also when all the dissipation of the system is carried out by the memory term solely, provided that an additive constraint on the so-called flatness rate of the kernel (cf. [18]) is assumed. Therefore, for the sake of our problem, we introduce the history space setting, which indeed enables us to achieve decay results removing differential inequalities on the kernel $k$. Nevertheless, a complete parallel to the second order problem [ 6 does not hold. That is, we are not yet able to treat the limiting case $\beta=0$, which seems to require the introduction of some new, deep technique. Thus, in this paper we shall work under the restriction $\beta>0$, with the 
only exception of the last section, where we shall in fact investigate polynomial stability assuming $k$ to fulfill a differential inequality.

Memory kernel hypotheses. We now briefly discuss some conditions and results already available in the literature. As the infinite-delay counterpart of (1.1) generates a linear semigroup, in [11] it is shown that, whenever $k(s)$ is smooth and $\mu(s)=-k^{\prime}(s)$ fulfills the differential inequality

$$
\mu^{\prime}(s)+\delta \mu(s) \leq 0, \quad s>0,
$$

for some positive $\delta$, then the semigroup is exponentially stable. It is immediate to see that (1.3) is equivalent to

$$
\mu(t+s) \leq e^{-\delta t} \mu(s), \quad s>0, t \geq 0 .
$$

On the other hand, (1.4) is weakened in [3, where the assumption (first introduced in [18])

$$
\mu(t+s) \leq C e^{-\delta t} \mu(s), \quad t \geq 0,
$$

for some $C \geq 1, \delta>0$ and for a.e. $s>0$, is shown to be necessary and sufficient. Since (1.5) implies the existence of $\gamma>0$ such that

$$
k^{\prime}(s)+\gamma k(s) \leq 0,
$$

for a.e. $s>0$, we have a necessary condition for the exponential decay of the semigroup in terms of the kernel $k$.

We recall that (1.1) is only a particular instance, obtained by taking null values of $u(t)$ for negative times, of the infinite delay equation, so that its energy $\mathcal{E}(t)$ decays whenever the semigroup does. Nevertheless, our aim is to take advantage of the Volterra finite time delay in order to study the stability under more general assumptions than (1.6). To this purpose, Fabrizio and Polidoro in [9] make use of the so-called exponential decay property

$$
\int_{0}^{\infty} k(s) e^{\delta s} d s<\infty
$$

for some $\delta>0$. They show the necessity of (1.7) for a particular exponential stability property (which we discuss in the sequel), under the further assumption of square summability for $k$, and for the concrete formulation (1.2).

There is indeed a gap between conditions (1.5)-(1.6) and (1.7), as we will show in Section 3 with a counterexample.

For what concerns polynomial stability, we introduce the polynomial decay property for the kernel $k$ :

$$
\int_{0}^{\infty}(1+s)^{r} k(s) d s<\infty, \quad r>0 .
$$

Again, in 9] a result concerning the necessity of such a condition for polynomial decay is established. We will return to these issues with some detail later in Section 3 ,

The main theorem. In the present paper, under some basic assumptions that guarantee well-posedness and energy dissipation, we will show that uniform decay properties for $\mathcal{E}(t)$ hold assuming (1.7) or (1.8). Our main result, which will be stated rigorously in Section 3. reads as follows: provided that the kernel $k$ satisfies the exponential (resp. polynomial) decay property, then problem (1.1) is exponentially (resp. polynomially) stable. 
Plan of the paper. We conclude our introduction by outlining the rest of the paper. In Section 2 we state in a precise way the basic assumptions on the memory kernel. Then we translate the problem in the so-called history space setting and recall from [3] a well-posedness result. Moreover, we establish some general decay properties. In Section 3, the main theorem is rigorously stated under some dissipativity assumptions on $k$ (corresponding to (1.7) and (1.8)), and the necessity of such assumptions is also discussed. Sections 4 and 5 are dedicated to proving the main result in the exponential and polynomial cases, respectively. Finally, in Section [6, the polynomial stability is revisited, under the further requirement that the kernel fulfills a differential inequality, even in the degenerate case $\beta=0$.

2. Semigroup generation and general decay properties. In order to proceed in our investigation, as already remarked, it is convenient to view our problem as an ordinary differential equation in a proper Hilbert space accounting for the past history of the variable $u$ (cf. [7). We first state the basic assumptions on the kernel, which are a refinement of the assumptions outlined in the introduction.

Basic assumptions. We suppose $k$ to be a (nonnegative) decreasing convex summable function, and we require its a.e. derivative $k^{\prime}$ to be summable as well. Let

$$
\mu(s)=-k^{\prime}(s)
$$

and

$$
\kappa=\int_{0}^{\infty} \mu(s) d s<\infty .
$$

Corner points for $k$ are allowed, but with the following restriction. We assume the set of jump points of $\mu$ to be a strictly increasing sequence $\left\{s_{n}\right\}_{n \geq 1} \in \mathbb{R}^{+}$, either finite (possibly empty) or converging to $s_{\infty} \in(0, \infty]$, such that $\mu$ is absolutely continuous on each interval $I_{n}=\left(s_{n}, s_{n+1}\right)$. If $s_{\infty}<\infty$, we also require the absolute continuity on the interval $\left(s_{\infty}, \infty\right)$, whereas in this case $s_{\infty}$ may or may not be a jump point.

Under such hypotheses, $\mu$ is decreasing and possibly singular for $s \rightarrow 0^{+}, \mu^{\prime}$ is defined almost everywhere, and $k$ is a piecewise $C^{1}$ function.

REMARK 2.1. In the rest of the paper, these basic assumptions are always understood to hold.

The history space setting. We extend the solution $u$ to negative times, setting $u(t)=0$ for $t<0$, and we introduce the following auxiliary past history variable:

$$
\eta^{t}(s)=\int_{t-s}^{t} u(r) d r, \quad t \geq 0, \quad s>0 .
$$

Note immediately that $\eta^{0}(s)=0$ for all $s>0$. According to the above definition, the integro-differential equation of problem (1.1) reads (cf. [3])

$$
\partial_{t} u(t)+\beta A u(t)+\int_{0}^{\infty} \mu(s) A \eta^{t}(s) d s=0, \quad t>0 .
$$


The past history variable $\eta$ is the unique mild solution (in the sense of [20, §4]) of an abstract Cauchy problem in the $\mu$-weighted space $\mathcal{M}=L_{\mu}^{2}\left(\mathbb{R}^{+}, V\right)$, that is,

$$
\left\{\begin{array}{l}
\partial_{t} \eta^{t}=T \eta^{t}+u(t), \quad t>0 \\
\eta^{0}=0
\end{array}\right.
$$

where, as a consequence of the basic assumptions (see [12]), the linear operator $T$ is the infinitesimal generator of the right-translation $C_{0}$-semigroup on $\mathcal{M}$, defined as

$$
T \eta=-\eta^{\prime}, \quad \mathcal{D}(T)=\left\{\eta \in \mathcal{M}: \eta^{\prime} \in \mathcal{M}, \lim _{s \rightarrow 0^{+}} \eta(s)=0 \text { in } V\right\} .
$$

Here the superposed prime symbol denotes the distributional derivative with respect to the internal variable $s$. Consequently, we define the Hilbert space

$$
\mathcal{H}=H \times \mathcal{M}
$$

normed by

$$
\|(u, \eta)\|_{\mathcal{H}}^{2}=\|u\|^{2}+\|\eta\|_{\mathcal{M}}^{2},
$$

with

$$
\|\eta\|_{\mathcal{M}}^{2}=\int_{0}^{\infty} \mu(s)\|\eta(s)\|_{V}^{2} d s .
$$

From now on, we let $u_{0}=u(0)$ and $\eta_{0}=\eta^{0}$.

In this setting (cf. [3, Section 3]) it is possible to show that a function $u$ is a weak solution to (1.1) if and only if the vector $z=(u, \eta)$ is a mild solution of the ordinary differential equation in $\mathcal{H}$,

$$
\left\{\begin{array}{l}
\frac{d}{d t} z(t)=\mathbb{L} z(t), \quad t>0 \\
z(0)=\left(u_{0}, 0\right) .
\end{array}\right.
$$

The operator $\mathbb{L}$ is defined by

$$
\begin{gathered}
\mathbb{L}(u, \eta)=\left(-A\left(\beta u+\int_{0}^{\infty} \mu(s) \eta(s) d s\right), T \eta+u\right) \\
\mathcal{D}(\mathbb{L})=\left\{z \in \mathcal{H}: u \in V, \beta u+\int_{0}^{\infty} \mu(s) \eta(s) d s \in \mathcal{D}(A), \eta \in \mathcal{D}(T)\right\} .
\end{gathered}
$$

Moreover, in our present case the explicit representation formula (cf. [19]) for $\eta$ reads

$$
\eta^{t}(s)= \begin{cases}\int_{0}^{s} u(t-r) d r, & 0<s \leq t, \\ \int_{0}^{t} u(t-r) d r, & s>t .\end{cases}
$$

As shown in [3, we have the following well-posedness result.

THEOREM 2.2. The equation in problem (2.1) generates a $C_{0}$-semigroup of contractions $S(t)$ on $\mathcal{H}$.

Remark 2.3. We stress that Theorem 2.2 holds on the whole space $\mathcal{H}$, not just on $H \times\{0\}$. 
As a consequence, for any given $\left(u_{0}, \eta_{0}\right) \in \mathcal{H}$, we have

$$
z(t)=\left(u(t), \eta^{t}\right)=S(t)\left(u_{0}, \eta_{0}\right) .
$$

The energy of the semigroup is defined as

$$
\mathbb{E}(t)=\mathbb{E}(z(t))=\left\|S(t)\left(u_{0}, \eta_{0}\right)\right\|_{\mathcal{H}}^{2} .
$$

Moreover (see [3] again), it follows

TheOrem 2.4. For every initial data $\left(u_{0}, \eta_{0}\right) \in \mathcal{D}(\mathbb{L})$, the solution $S(t)\left(u_{0}, \eta_{0}\right)$ belongs to $C^{1}([0, \infty), \mathcal{D}(\mathbb{L}))$, and the associated energy fulfills the differential identity

$$
\frac{d}{d t} \mathbb{E}(t)+2 \beta\|u(t)\|_{V}^{2}-\int_{0}^{\infty} \mu^{\prime}(s)\left\|\eta^{t}(s)\right\|_{V}^{2} d s+\mathbb{J}\left[\eta^{t}\right]=0,
$$

with

$$
\mathbb{J}\left[\eta^{t}\right]=\sum_{n}\left[\mu\left(s_{n}^{-}\right)-\mu\left(s_{n}^{+}\right)\right]\left\|\eta^{t}\left(s_{n}\right)\right\|_{V}^{2},
$$

where the sum includes the value $n=\infty$ if $s_{\infty}<\infty$. In particular, $\mathbb{E}(t)$ is a decreasing function of $t$.

From now on we shall restrict our investigation to initial data of the form $\left(u_{0}, 0\right)$, to take advantage of the one-to-one correspondence between problems (1.1) and (2.1). Also, the associated energy is

$$
\mathcal{E}(t)=\left\|S(t)\left(u_{0}, 0\right)\right\|_{\mathcal{H}}^{2} .
$$

In view of the representation formula (2.2), this is indeed the energy defined in the introduction. Since an initial datum of the form $\left(u_{0}, 0\right) \in H \times\{0\}$ belongs to $\mathcal{D}(\mathbb{L})$ if $u_{0} \in V$, it follows that $\mathcal{E}(t)$ satisfies equality (2.3) for any $u_{0} \in V$.

REMARK 2.5. In the sequel, we shall always assume the initial datum to belong to $V \times$ $\{0\}$. Thanks to the semigroup continuity property, our decay results will be understood to extend by density to the whole space $H \times\{0\}$, where equality (2.3) is still fulfilled, provided that the time derivative of $\mathcal{E}(t)$ is intended in the sense of distributions.

General decay properties. We first notice that, without introducing new assumptions, it is possible to obtain a uniform decay for the energy. To this purpose, define

$$
U(t)=\int_{0}^{t} u(y) d y
$$

so that (2.2) turns out to be

$$
\eta^{t}(s)=U(t)-U(t-s), \quad t \geq 0, s>0,
$$

where it is understood that $U(t)=0$ for $t \leq 0$. We have the following

THEOREM 2.6. $\mathcal{E}(t)$ is polynomially stable of rate 1.

Proof. We introduce the following energy functionals, already encountered in [3]:

$$
\begin{aligned}
& \Psi_{1}(t)=\frac{1}{2}\langle U(t), u(t)\rangle, \\
& \Psi_{2}(t)=\frac{1}{2} \int_{0}^{\infty} k(s)\left\|\eta^{t}(s)-U(t)\right\|_{V}^{2} d s .
\end{aligned}
$$


These functionals are well defined since (2.4) holds and $U(t-s)=0$ for $s \geq t$. Due to the continuity of $k(s)$, there exist $\bar{s}>0, C \geq 0$ such that $k(s) \leq C \mu(s)$ for all $s \in(0, \bar{s}]$. Then

$$
\begin{aligned}
\int_{0}^{\bar{s}} k(s)\|U(t)\|_{V}^{2} d s & \leq 2 \int_{0}^{\bar{s}} k(s)\left\|\eta^{t}(s)\right\|_{V}^{2} d s+2 \int_{0}^{\bar{s}} k(s)\|U(t-s)\|_{V}^{2} d s \\
& \leq 2 C \int_{0}^{\bar{s}} \mu(s)\left\|\eta^{t}(s)\right\|_{V}^{2} d s+2 \int_{0}^{\bar{s}} k(s)\|U(t-s)\|_{V}^{2} d s \\
& \leq 2 C\left\|\eta^{t}\right\|_{\mathcal{M}}^{2}+2 \Psi_{2}(t),
\end{aligned}
$$

so, letting $Q=2\left(\int_{0}^{\bar{s}} k(s) d s\right)^{-1} \max \{1, C\}$ we end up with

$$
\|U(t)\|_{V}^{2} \leq Q\left[\left\|\eta^{t}\right\|_{\mathcal{M}}^{2}+\Psi_{2}(t)\right] .
$$

A straightforward computation (see [3]) shows that $\Psi(t)=2 \Psi_{1}(t)+\Psi_{2}(t)$ fulfills the differential equality

$$
\frac{d}{d t} \Psi(t)=-\frac{1}{2}\left\|\eta^{t}\right\|_{\mathcal{M}}^{2}+\|u(t)\|^{2}
$$

We now define, for $M>0$, the further functional

$$
\mathcal{J}(t)=M \mathcal{E}(t)+\Psi(t) .
$$

Therefore, recalling the energy identity (2.3) and the Poincaré inequality $\lambda\|\cdot\|^{2} \leq\|\cdot\|_{V}^{2}$ (corresponding to the continuous inclusion $V \hookrightarrow H$ ), we obtain, for $M$ large enough,

$$
\frac{d}{d t} \mathcal{J}(t)+\varepsilon \mathcal{E}(t) \leq 0
$$

for some $\varepsilon>0$. Since $\eta^{0}(s) \equiv 0$, we have $\mathcal{J}(0)=M \mathcal{E}(0)$. Integrating the above inequality on $(0, t)$ we get

$$
\mathcal{J}(t)+\varepsilon \int_{0}^{t} \mathcal{E}(\tau) d \tau \leq M \mathcal{E}(0)
$$

Then

$$
\Psi(t)+\varepsilon \int_{0}^{t} \mathcal{E}(\tau) d \tau \leq M \mathcal{E}(0)
$$

and

$$
\Psi_{2}(t)+\varepsilon \int_{0}^{t} \mathcal{E}(\tau) d t \leq M \mathcal{E}(0)-\langle U(t), u(t)\rangle \leq M \mathcal{E}(0)+\|U(t)\| \sqrt{\mathcal{E}(0)} .
$$

By means of (2.5), with the Young and Poincaré inequalities we have

$$
\|U(t)\| \sqrt{\mathcal{E}(0)} \leq \frac{Q}{4 \lambda} \mathcal{E}(0)+\left\|\eta^{t}\right\|_{\mathcal{M}}^{2}+\Psi_{2}(t) .
$$

Letting $K=\varepsilon^{-1}\left(1+M+\frac{Q}{4 \lambda}\right)$ we obtain

$$
\int_{0}^{t} \mathcal{E}(\tau) d \tau \leq K \mathcal{E}(0)
$$


In particular, $\mathcal{E}(t)$ being decreasing, we conclude

$$
t \mathcal{E}(t) \leq \int_{0}^{t} \mathcal{E}(\tau) d \tau
$$

so that

$$
\mathcal{E}(t) \leq \frac{K \mathcal{E}(0)}{t} .
$$

Since $\mathcal{E}(t)$ is bounded, the thesis follows.

REMARK 2.7. Changing the constants in the Young inequality used in (2.8), we find

$$
\|U(t)\| \sqrt{\mathcal{E}(0)} \leq \frac{Q}{2 \lambda} \mathcal{E}(0)+\frac{1}{2}\left\|\eta^{t}\right\|_{\mathcal{M}}^{2}+\frac{1}{2} \Psi_{2}(t),
$$

so from (2.7) it is easily seen that, letting $R$ be a generic positive constant, $\Psi_{2}(t) \leq R \mathcal{E}(0)$. This holds also for $\|U(t)\|_{V}^{2}$, thanks to (2.5). Moreover, back to (2.4), we infer

$$
\left\|\eta^{t}(s)\right\|_{V}^{2} \leq R \mathcal{E}(0)
$$

uniformly in $t$ and $s$. This is the main consequence of the Volterra equation finite delay structure, and in the sequel it will play an important role.

3. The main result. We will state our main result under the following reformulation of conditions (1.7) and (1.8).

Dissipativity assumptions. Let $p \in(1, \infty]$. Suppose there exists $C \geq 0$ such that

$$
k(s) \leq C \Lambda_{p}(s), \quad s>0,
$$

where

$$
\Lambda_{p}(s)= \begin{cases}e^{-\delta s}(\delta>0), & \text { if } p=\infty ; \\ \frac{1}{(1+s)^{p}}, & \text { if } p<\infty .\end{cases}
$$

Notice that, $k$ being a decreasing function, (3.1) with $p=\infty$ is equivalent to the exponential decay property (1.7), up to replacing $\delta$ in (1.7) with an arbitrarily chosen $\gamma>\delta$. Also, the polynomial decay property (1.8) implies (3.1) with $p=r+1$. Conversely, if (3.1) holds for $p \in(1, \infty)$, (1.8) follows with $r=p-1-\varepsilon$, for any (small) $\varepsilon>0$. Therefore, we have the correspondence between (3.1) and (1.7)-(1.8).

REMARK 3.1. Condition (3.1) with $p=\infty$ is weaker than the differential inequality (1.6), even for convex kernels. A counterexample can be constructed as follows. For $n \in \mathbb{N}$, define

$$
k_{n}(s)=-e^{-n^{2}} s+\left(1+n^{2}\right) e^{-n^{2}} .
$$

Then the kernel

$$
k(s)=\max _{n \in \mathbb{N}} k_{n}(s)
$$

fulfills (3.1) for $p=\infty$, with $C=\delta=1$, but easy computations show that (1.6) cannot hold.

We immediately prove a lemma which yields a useful estimate to be used in the sequel. 
Lemma 3.2. Let $k$ enjoy the dissipativity condition (3.1). Then, for any $\omega \in(1 /(p+1), 1)$ there exists a positive $c=c(p, \omega, \kappa)$ such that

$$
\int_{0}^{\infty}[\mu(s)]^{\omega} d s \leq c<\infty .
$$

Proof. Since $\mu$ is decreasing, (3.1) can be equivalently stated as follows. There exist $C \geq 0$ and $s_{*} \geq 0$ such that

$$
\mu(s) \leq C \Lambda_{p+1}(s), \quad s>s_{*},
$$

where $s_{*}$ can be chosen to be zero if $\mu$ is not singular at the origin. Let

$$
\mathcal{A}=\left\{s \in\left(0, s_{*}\right): \mu(s)>1\right\} \quad \text { and } \quad \mathcal{B}=\left\{s \in\left(0, s_{*}\right): \mu(s) \leq 1\right\} .
$$

Then, exploiting (3.2), we have

$$
\int_{0}^{\infty}[\mu(s)]^{\omega} d s=\int_{\mathcal{A}}[\mu(s)]^{\omega} d r+\int_{\mathcal{B}}[\mu(s)]^{\omega} d s+\int_{s_{*}}^{\infty}[\mu(s)]^{\omega} d s \leq \kappa+s_{*}+C^{\omega} c_{*},
$$

where

$$
c_{*}=\int_{s_{*}}^{\infty}\left[\Lambda_{p+1}(s)\right]^{\omega} d s= \begin{cases}\frac{e^{\omega \delta s_{*}}}{\omega \delta}, & \text { if } p=\infty ; \\ \frac{\left(1+s_{*}\right)^{-\omega(p+1)+1}}{\omega(p+1)-1}, & \text { if } p<\infty .\end{cases}
$$

The thesis is then achieved, provided that we choose $c=\kappa+s_{*}+C^{\omega} c_{*}$.

REMARK 3.3. We stress that, in the case $p=\infty$, the lower bound on $\omega$ in the lemma above is to be considered as 0 . Moreover, in this case, the constant $c$ depends on $\delta$ also.

We now state the main result of this paper.

Theorem 3.4. Assume condition (3.1). Then

- if $p=\infty$, then $\mathcal{E}(t)$ is exponentially stable;

- if $p \in(2, \infty)$, then $\mathcal{E}(t)$ is polynomially stable of rate $r$, for any $r<p-1$.

Necessary conditions. Before proceeding with the proof, we discuss the main result in comparison with the necessary conditions obtained by Fabrizio and Polidoro (see [9]) and mentioned in the introduction. Indeed, in [9], for the particular case of the concrete formulation (1.2), the authors proved (by means of Laplace transform methods) that if the solution $u$ satisfies

$$
\int_{0}^{\infty} e^{\alpha t}\|u(t)\|_{V}^{2} d t<\infty
$$

for some $\alpha>0$ and the kernel $k$ is square integrable, then (1.7) holds. To be consistent, until the end of this section, we restrict ourselves to formulation (1.2), and we also assume $\sqrt{k} \in L^{1}\left(\mathbb{R}^{+}\right)$. Then, condition (3.1) with $p=\infty$ is easily shown to be necessary as well. In fact, starting from an exponentially decaying energy

$$
\mathcal{E}(t) \leq \mathcal{Q} e^{-\varepsilon t},
$$

and recalling identity (2.3), we have

$$
\int_{0}^{t} e^{\gamma \tau} \frac{d}{d t} \mathcal{E}(\tau) d \tau+2 \beta \int_{0}^{t} e^{\gamma \tau}\|u(\tau)\|_{V}^{2} d \tau \leq 0
$$


for some fixed $\gamma>0$. An integration by parts yields

$$
e^{\gamma t} \mathcal{E}(t)-\mathcal{E}(0)-\gamma \int_{0}^{t} e^{\gamma \tau} \mathcal{E}(\tau) d \tau+2 \beta \int_{0}^{t} e^{\gamma \tau}\|u(\tau)\|_{V}^{2} d \tau \leq 0,
$$

so that condition (3.3) is fulfilled, provided that we choose $\gamma<\varepsilon$. The exponential decay property for $k$ follows by [9, Theorem 2.5].

Concerning polynomial stability, we first recall the necessary condition of [9]: defining

$$
\begin{aligned}
& p_{0}=\sup \left\{r \geq 0: \int_{0}^{\infty}(1+s)^{r-1} k(s) d s<\infty\right\}, \\
& q_{0}=\sup \left\{r \geq 0: \int_{0}^{\infty}(1+t)^{2 r-1}\|u(t)\|_{V}^{2} d t<\infty\right\},
\end{aligned}
$$

result [9. Theorem 4.2] reads $p_{0} \geq q_{0}$ under the hypothesis $q_{0}>1$. That is, if

$$
\sup \left\{r \geq 0: \int_{0}^{\infty}(1+t)^{r}\|u(t)\|_{V}^{2} d t<\infty\right\}=p
$$

for some $p>1$, then

$$
\sup \left\{r \geq 0: \int_{0}^{\infty}(1+s)^{r} k(s) d s<\infty\right\} \geq \frac{p-1}{2} .
$$

REMARK 3.5. Such a necessary condition, as remarked also in [6], is somehow unsatisfactory, although difficult to improve. Indeed, for large values of $p$ the rate loss is rather gross.

Now let $p>1$ and

$$
\mathcal{E}(t) \leq \frac{\mathcal{Q}}{(1+t)^{p}}
$$

We can show how (3.5) is still necessary. To this purpose, introduce $\gamma \in(0, p)$; back to (2.3) once again, we are led to

$$
\int_{0}^{t}(1+\tau)^{\gamma} \frac{d}{d t} \mathcal{E}(\tau) d \tau+2 \beta \int_{0}^{t}(1+\tau)^{\gamma}\|u(\tau)\|_{V}^{2} d \tau \leq 0
$$

so that, by means of an integration by parts, we deduce

$$
(1+t)^{\gamma} \mathcal{E}(t)-\mathcal{E}(0)-\gamma \int_{0}^{t}(1+\tau)^{\gamma-1} \mathcal{E}(\tau) d \tau+2 \beta \int_{0}^{t}(1+\tau)^{\gamma}\|u(\tau)\|_{V}^{2} d \tau \leq 0
$$

The first three summands are bounded, so

$$
\int_{0}^{\infty}(1+\tau)^{\gamma}\|u(\tau)\|_{V}^{2} d \tau<\infty
$$

for any $\gamma<p$. Then, (3.4) holds, and we can apply the result of [9] to obtain (3.5).

Summing up, we have proved the following.

Theorem 3.6. Assume (1.2) to hold and $\sqrt{k} \in L^{1}\left(\mathbb{R}^{+}\right)$.

- If $\mathcal{E}(t)$ is exponentially stable, then (3.1) holds with $p=\infty$.

- If $\mathcal{E}(t)$ is polynomially stable of rate $r>1$, then (3.1) holds for any $p<\frac{r+1}{2}$. 
4. Exponential stability. In this section we will prove Theorem 3.4 in the case $p=\infty$. We begin by defining the following functionals:

$$
\begin{aligned}
& \Upsilon(t)=\int_{0}^{\infty} k(s)\left\|\eta^{t}(s)\right\|_{V}^{2} d s, \\
& \Theta(t)=\int_{0}^{\infty} e^{-\delta s}\left\|\eta^{t}(s)\right\|_{V}^{2} d s,
\end{aligned}
$$

$\delta$ being as in (3.1). The next set of lemmas provides essential estimates involving the above functionals, which will be crucial in the sequel. For the rest of this paper, let $c$ be a (positive) generic constant.

LEmma 4.1. The functionals $\Upsilon$ and $\Theta$ are well defined and fulfill the inequality

$$
\Upsilon(t) \leq c \Theta(t) .
$$

Proof. Inequality (4.1) follows directly by assumption (3.1). Moreover, using the boundedness of $\left\|\eta^{t}(s)\right\|_{V}$ (see Remark 2.7), the well-definedness of $\Theta$ is achieved, whereas the one for $\Upsilon$ is a consequence of (4.1).

LEmma 4.2. The following differential inequalities hold:

$$
\begin{gathered}
\frac{d}{d t} \Upsilon(t)+\frac{1}{2}\left\|\eta^{t}\right\|_{\mathcal{M}}^{2} \leq c\|u(t)\|_{V}^{2}, \\
\frac{d}{d t} \Theta(t)+\frac{\delta}{2} \Theta(t) \leq c\|u(t)\|_{V}^{2} .
\end{gathered}
$$

Proof. Notice previously that, as $\mu$ is nonincreasing,

$$
\int_{s}^{\infty} \mu(r) d r=\int_{s}^{\infty}[\mu(r)]^{2 / 3}[\mu(r)]^{1 / 3} d r \leq[\mu(s)]^{2 / 3} \int_{0}^{\infty}[\mu(r)]^{1 / 3} d r
$$

for any $s>0$. Therefore, choosing $\omega=1 / 3$ in Lemma 3.2. we are led to

$$
k(s) \leq c[\mu(s)]^{2 / 3}, \quad s>0 .
$$

By means of the equation for the past history variable, a direct calculation leads to the following differential equality for $\Upsilon(t)$ :

$$
\frac{d}{d t} \Upsilon(t)+\left\|\eta^{t}\right\|_{\mathcal{M}}^{2}=2 \int_{0}^{\infty} k(s)\left\langle\eta^{t}(s), u(t)\right\rangle_{V} d s
$$

Concerning the right-hand side, we have

$$
\int_{0}^{\infty} k(s)\left\langle\eta^{t}(s), u(t)\right\rangle_{V} \leq \nu\left(\int_{0}^{\infty} k(s)\left\|\eta^{t}(s)\right\|_{V} d s\right)^{2}+\frac{1}{\nu}\|u(t)\|_{V}^{2},
$$

for some $\nu \in(0,1)$. As a consequence of (4.4) and again Lemma 3.2 with $\omega=1 / 3$, we get

$$
\begin{aligned}
\int_{0}^{\infty} k(s)\left\|\eta^{t}(s)\right\|_{V} d s & \leq c \int_{0}^{\infty}[\mu(s)]^{2 / 3}\left\|\eta^{t}(s)\right\|_{V} d s \\
& \leq c\left(\int_{0}^{\infty}[\mu(s)]^{1 / 3} d s\right)^{1 / 2}\left\|\eta^{t}\right\|_{\mathcal{M}} \leq c\left\|\eta^{t}\right\|_{\mathcal{M}} .
\end{aligned}
$$


Therefore

$$
2 \int_{0}^{\infty} k(s)\left\langle u(t), \eta^{t}(s)\right\rangle_{V} \leq 2 \nu c^{2}\left\|\eta^{t}\right\|_{\mathcal{M}}^{2}+\frac{2}{\nu}\|u(t)\|_{V}^{2},
$$

which, back to equality (4.5), yields (4.2), provided that we choose $\nu$ small enough. Analogously to (4.5), we see that $\Theta(t)$ fulfills the differential equality

$$
\frac{d}{d t} \Theta(t)+\delta \Theta(t)=2 \int_{0}^{\infty} e^{-\delta t}\left\langle\eta^{t}(s), u(t)\right\rangle_{V} d s .
$$

It is immediate to realize that

$$
\int_{0}^{\infty} e^{-\delta t}\left\langle\eta^{t}(s), u(t)\right\rangle_{V} d s \leq \frac{\delta}{4} \Theta(t)+\frac{1}{\delta}\left(\int_{0}^{\infty} e^{-\delta s} d s\right)\|u(t)\|_{V}^{2},
$$

which, in (4.6), concludes the proof.

We end the section with the proof of the exponential stability result.

Proof of Theorem 3.4 (case $p=\infty$ ). We define the additional functional

$$
\mathcal{L}(t)=M \mathcal{E}(t)+\Upsilon(t)+\Theta(t),
$$

for some $M \geq 1$ to be suitably chosen in the sequel. Inequality (4.1) implies that

$$
\mathcal{E}(t)+\Theta(t) \leq \mathcal{L}(t) \leq c[\mathcal{E}(t)+\Theta(t)] .
$$

Summing up inequalities (2.3), (4.2) and (4.3), and recalling the Poincaré inequality, we deduce

$$
\frac{d}{d t} \mathcal{L}(t)+M \beta \lambda\|u(t)\|^{2}+\frac{1}{2}\left\|\eta^{t}\right\|_{\mathcal{M}}^{2}+\frac{\delta}{2} \Theta(t)+(M \beta-c)\|u(t)\|_{V}^{2} \leq 0 .
$$

Provided that $M$ is large enough, by setting $\varepsilon=c^{-1} \min \{M \beta \lambda, 1 / 2, \delta / 2\}$, we finally reach

$$
\frac{d}{d t} \mathcal{L}(t)+\varepsilon \mathcal{L}(t) \leq 0
$$

By means of (4.7), invoking the Gronwall Lemma, we get

$$
\mathcal{E}(t) \leq \mathcal{L}(t) \leq M \mathcal{E}(0) e^{-\varepsilon t}=\mathcal{Q} e^{-\varepsilon t},
$$

which concludes the proof.

5. Polynomial stability. In this section we will prove Theorem 3.4 in the case $p \in(2, \infty)$.

REMARK 5.1. For the case $p \leq 2$ we refer the reader to Theorem 2.6.

Again, the proof requires the introduction of suitable functionals. We let $\Upsilon$ be as in the previous section; also, we define

$$
\Theta_{p}(t)=\int_{0}^{\infty} \frac{1}{(1+s)^{p}}\left\|\eta^{t}(s)\right\|_{V}^{2} d s
$$

Analogously to the case $p=\infty$, we have 
LEmma 5.2. The functionals $\Upsilon$ and $\Theta_{p}$ are well defined and fulfill the inequalities

$$
\Upsilon(t) \leq c \Theta_{p}(t) \leq C_{q}\left(\int_{0}^{\infty} \frac{1}{(1+s)^{p+1}}\left\|\eta^{t}(s)\right\|_{V}^{2} d s\right)^{1 / q}
$$

for any $q>p /(p-1)$, where $C_{q}$ is a positive constant depending only on $q$ and (increasingly) on $\mathcal{E}(0)$.

Proof. The left-hand side of inequality (5.1) follows directly by assumption (3.1). By means of the Hölder inequality, we have, for some $0<\sigma<p$,

$$
\begin{aligned}
\Theta_{p}(t) & =\int_{0}^{\infty} \frac{1}{(1+s)^{\sigma}} \frac{1}{(1+s)^{p-\sigma}}\left\|\eta^{t}(s)\right\|_{V}^{2} d s \\
& \leq\left(\int_{0}^{\infty} \frac{1}{(1+s)^{\sigma(p+1) /(1+\sigma)}}\left\|\eta^{t}(s)\right\|_{V}^{2} d s\right)^{\frac{1+\sigma}{p+1}}\left(\int_{0}^{\infty} \frac{1}{(1+s)^{p+1}}\left\|\eta^{t}(s)\right\|_{V}^{2} d s\right)^{\frac{p-\sigma}{p+1}} .
\end{aligned}
$$

By virtue of Remark 2.7, we have $\left\|\eta^{t}(s)\right\|_{V}^{2} \leq \mathcal{Q}$, so the first factor is well defined for $\sigma>1 / p$. Therefore

$$
\left[\Theta_{p}(t)\right]^{q} \leq C_{q}^{q} \int_{0}^{\infty} \frac{1}{(1+s)^{p+1}}\left\|\eta^{t}(s)\right\|_{V}^{2} d s
$$

for $q>p /(p-1)$, since

$$
C_{q}=\left(\int_{0}^{\infty} \frac{1}{(1+s)^{(p q-p-1) /(q-1)}} d s\right)^{\frac{q-1}{q}} \mathcal{Q} .
$$

This proves the right-hand side inequality in (5.1). Moreover, by the uniform bound on $\left\|\eta^{t}(s)\right\|_{V}$, we infer the well-definedness of $\Theta_{p}(t)$, and as a consequence of the left-hand side inequality in (5.1), also of $\Upsilon(t)$.

REMARK 5.3. It is worth pointing out that the constant $C_{q}$ above is well defined only for $q>p /(p-1)$ and tends to infinity for $q \downarrow p /(p-1)$.

Lemma 5.4. The following differential inequalities hold:

$$
\begin{aligned}
& \frac{d}{d t} \Upsilon(t)+\frac{1}{2}\left\|\eta^{t}\right\|_{\mathcal{M}}^{2} \leq c\|u(t)\|_{V}^{2}, \\
& \frac{d}{d t} \Theta_{p}(t)+k_{q}\left[\Theta_{p}(t)\right]^{q} \leq c\|u(t)\|_{V}^{2},
\end{aligned}
$$

for any $q>p /(p-1)$, choosing $k_{q}=C_{q}^{-q}$.

Proof. The proof of inequality (5.3) goes exactly like the one of (4.2), noticing that $\omega$ in Lemma 3.2 can be chosen to be $1 / 3$ for all $p>2$. Concerning (5.4), a straightforward computation shows that

$$
\frac{d}{d t} \Theta_{p}(t)+p \int_{0}^{\infty} \frac{1}{(1+s)^{p+1}}\left\|\eta^{t}(s)\right\|_{V}^{2} d s=2 \int_{0}^{\infty} \frac{1}{(1+s)^{p}}\left\langle\eta^{t}(s), u(t)\right\rangle_{V} d s .
$$


Again by the Hölder inequality we infer

$$
\begin{aligned}
& \int_{0}^{\infty} \frac{1}{(1+s)^{p}}\left\langle\eta^{t}(s), u(t)\right\rangle_{V} d s \\
\leq & \int_{0}^{\infty} \frac{1}{(1+s)^{(p+1) / 2}} \frac{1}{(1+s)^{(p-1) / 2}}\left\|\eta^{t}(s)\right\|_{V}\|u(t)\|_{V} d s \\
\leq & \left(\int_{0}^{\infty} \frac{1}{(1+s)^{p+1}}\left\|\eta^{t}(s)\right\|_{V}^{2} d s\right)^{1 / 2}\left(\int_{0}^{\infty} \frac{1}{(1+s)^{p-1}}\|u(t)\|_{V}^{2} d s\right)^{1 / 2} \\
\leq & \frac{p}{2} \int_{0}^{\infty} \frac{1}{(1+s)^{p+1}}\left\|\eta^{t}(s)\right\|_{V}^{2} d s+\frac{1}{2 p}\left(\int_{0}^{\infty} \frac{1}{(1+s)^{p-1}} d s\right)\|u(t)\|_{V}^{2} .
\end{aligned}
$$

Notice that, as $p>2$, the last term is well defined. Therefore, from (5.5) we deduce the inequality

$$
\frac{d}{d t} \Theta_{p}(t)+\frac{p}{2} \int_{0}^{\infty} \frac{1}{(1+s)^{p+1}}\left\|\eta^{t}(s)\right\|_{V}^{2} d s \leq c\|u(t)\|_{V}^{2} .
$$

By means of the right-hand side inequality in (5.1), this will imply (5.4), provided that we choose $k_{q}=C_{q}^{-q}$.

REMARK 5.5. As a consequence of Remark 5.3, the constant $k_{q}$ defined above tends to 0 for $q \downarrow p /(p-1)$.

Again, we end the section with the proof of the polynomial stability result.

Proof of Theorem 3.4 (case $2<p<\infty$ ). We define, for $M \geq 1$, the functional

$$
\mathcal{L}_{p}(t)=M \mathcal{E}(t)+\Upsilon(t)+\Theta_{p}(t) .
$$

Inequality (5.1) implies that

$$
\mathcal{E}(t)+\Theta_{p}(t) \leq \mathcal{L}_{p}(t) \leq c\left[\mathcal{E}(t)+\Theta_{p}(t)\right] .
$$

Let $q$ be fixed, with $q>p /(p-1)$. Collecting the differential inequalities (2.3), (5.3) and (5.4), we are led to

$$
\frac{d}{d t} \mathcal{L}_{p}(t)+M \beta \lambda\|u(t)\|^{2}+\frac{1}{2}\left\|\eta^{t}\right\|_{\mathcal{M}}^{2}+k_{q}[\Theta(t)]^{q}+(M \beta-c)\|u(t)\|_{V}^{2} \leq 0,
$$

where $\lambda$ is the constant appearing in the Poincaré inequality. $q$ being fixed, we have $C_{q} \leq \mathcal{Q}$ and $k_{q} \geq \mathcal{Q}^{-1}$. Therefore, provided that $M$ is large enough, we deduce

$$
\frac{d}{d t} \mathcal{L}_{p}(t)+\frac{1}{\mathcal{Q}}\left\{\mathcal{E}(t)+\left[\theta_{p}(t)\right]^{q}\right\} \leq 0 .
$$

Since $\Upsilon(t) \leq c \Theta_{p}(t)$, and since $\mathcal{E}(t) \leq \mathcal{E}(0) \leq \mathcal{Q}$, we have, for any $q>1$,

$$
\left[\mathcal{L}_{p}(t)\right]^{q} \leq \mathcal{Q}\left\{\mathcal{E}(t)+\left[\Theta_{p}(t)\right]^{q}\right\}
$$

so that, by the differential inequality above, we eventually end up with

$$
\frac{d}{d t} \mathcal{L}_{p}(t)+\frac{1}{\mathcal{Q}}\left[\mathcal{L}_{p}(t)\right]^{q} \leq 0,
$$

which, by means of (5.7), implies the estimate

$$
\mathcal{E}(t) \leq \mathcal{L}_{p}(t) \leq \mathcal{Q}(1+t)^{-r} .
$$


From the limitation $q>p /(p-1)$, we immediately infer $r<p-1$, and we stress that the constant $\mathcal{Q}$ depends on $q$ and diverges for $q \downarrow p /(p-1)$.

REMARK 5.6. If the kernel has the form $1 /(1+s)^{r}$, condition (3.1) is verified with $p=r$, so we find a polynomial decay of the energy of rate $r-1-\varepsilon$; we also have the constraint $r>2$.

6. Polynomial decay for a kernel satisfying a differential inequality. Another task of our investigation is the polynomial stability whenever the kernel fulfills a differential inequality of the type

$$
-k^{\prime \prime}(s)+\delta\left[-k^{\prime}(s)\right]^{\omega} \leq 0, \quad s>0,
$$

for some $\delta>0$ and $\omega>1$. Some results under assumptions of this kind can be found in [1, 15, 16, 17] for the linear second order problem in viscoelasticity.

REMARK 6.1. The techniques exploited in this note are not fit to tackle the more difficult case of problem (1.1) with $\beta=0$. Indeed, in that case, the dissipation is due to the memory term solely. Nevertheless, under hypothesis (6.1), we are able to provide a result both for $\beta>0$ and $\beta=0$. We also mention that in the latter situation, problem (1.1) is an abstract version of the so-called Gurtin-Pipkin model for heat conduction (see [13]). Also, in the special case of an exponential kernel $k(s)=e^{-s}$, from the GurtinPipkin law we recover the Maxwell-Cattaneo model (cf. [2]).

We point out that, also for the case $\beta=0$, we have the correspondence between problems (1.1) and (2.1). Moreover, under the basic assumptions of Section 2, Theorem 2.2 still holds and the energy equality is given again by (2.3).

It is convenient to state (6.1) in terms of $\mu$, namely

$$
\mu^{\prime}(s)+\delta[\mu(s)]^{\omega} \leq 0, \quad s>0 ;
$$

this is the polynomial corresponding to the exponential condition (1.3). We will show that a stronger result than Theorem 3.4 can be provided under (6.2). Namely, the rate of decay is improved by one. Let us give the precise statement.

Theorem 6.2. Let $\beta \geq 0$ in problem (1.1). For any fixed $p>2$ assume that $\mu$ fulfills, for some $\delta>0$, the differential inequality

$$
\mu^{\prime}(s)+\delta[\mu(s)]^{\frac{p+1}{p}} \leq 0, \quad s>0 .
$$

Then $\mathcal{E}(t)$ is polynomially stable with rate $r$, for any $r<p-1$.

Notice immediately that assumption (6.3) implies that there exist $C \geq 0$ and $s_{*} \geq 0$ such that

$$
\mu(s) \leq \frac{C}{(1+s)^{p}}, \quad s>s_{*},
$$

holds. Therefore, arguing as in Lemma 3.2 , we easily see that

$$
\int_{0}^{\infty}[\mu(s)]^{\omega} d s<\infty
$$

for any $\omega \in(1 / p, 1)$. 
The proof of the case $\beta=0$ requires the introduction of the additional functional

$$
\Phi(t)=-\frac{1}{\kappa} \int_{0}^{\infty} \psi(s)\left\langle u(t), \eta^{t}(s)\right\rangle d s .
$$

Here, in order to handle the (possible) integrable singularity of $\mu$ at 0 , following [18, we have defined, for any fixed $s_{\nu} \in\left(0, s_{1}\right)$, the function $\psi=\psi_{s_{\nu}}:(0, \infty) \rightarrow[0, \infty)$, as

$$
\psi(s)=\mu\left(s_{\nu}\right) \chi_{\left(0, s_{\nu}\right]}(s)+\mu(s) \chi_{\left[s_{\nu}, \infty\right)}(s),
$$

where $\chi_{\mathrm{I}}$ denotes the indicator function of any interval $\mathrm{I} \subset(0, \infty)$.

REMARK 6.3. In order to recover Theorem 2.6 in the case $\beta=0$, we need to introduce an hypothesis on the so-called flatness rate of the kernel (see [3, 6]; see also [18]). One defines (for any measurable $\mathcal{P} \subset \mathbb{R}^{+}$) the probability measure

$$
m_{\mu}(\mathcal{P})=\frac{1}{\kappa} \int_{\mathcal{P}} \mu(s) d s,
$$

and the flatness set of $\mu$ as

$$
\mathcal{F}_{\mu}=\left\{s \in \mathbb{R}^{+}: \mu(s)>0, \mu^{\prime}(s)=0\right\} ;
$$

the flatness rate of $\mu$ is the quantity

$$
\mathcal{R}_{\mu}=m_{\mu}\left(\mathcal{F}_{\mu}\right) .
$$

If $\beta=0$, the functional $\mathcal{J}$ defined in the proof of Theorem 2.6 does not fulfill (2.6). Nevertheless, in [3] it is shown that the modified functional

$$
\mathcal{J}_{1}(t)=M \mathcal{E}(t)+\Phi(t)+a \Psi(t),
$$

where $M$ and $a$ are suitable positive constants, does satisfy (2.6), under the additional restriction $\mathcal{R}_{\mu}<1 / 2$. If such a condition holds, since $\mathcal{J}_{1}(0)=\mathcal{J}(0)$, we can integrate (2.6) and repeat the conclusion of the proof of Theorem 2.6 with $\mathcal{J}_{1}$ in place of $\mathcal{J}$. Now, assuming (6.3), it is easily seen that $\mathcal{R}_{\mu}=0$, so that Theorem 2.6 holds. In particular we get $\left\|\eta^{t}(s)\right\|_{V} \leq \mathcal{Q}$ (see Remark 2.7).

The next lemma provides a basic estimate on the derivative of $\Phi$.

Lemma 6.4. Let $\beta=0$ and (6.3) hold. Then, for every $\nu \in(0,1)$, there exist $s_{\nu} \in\left(0, s_{1}\right)$ and positive constants $c_{1}$ and $c_{2}$ depending only on $\nu$ (possibly unbounded as $\nu \rightarrow 0$ ), such that the functional $\Phi(t)$ fulfills the inequality

$$
\begin{aligned}
\frac{d}{d t} \Phi(t) \leq & -(1-\nu)\|u(t)\|^{2}+c_{1} \int_{0}^{\infty}[\mu(s)]^{\frac{p+1}{p}}\left\|\eta^{t}(s)\right\|_{V}^{2} d s \\
& -c_{2}\left(\int_{0}^{\infty} \mu^{\prime}(s)\left\|\eta^{t}(s)\right\|_{V}^{2} d s-\mathbb{J}\left[\eta^{t}\right]\right) .
\end{aligned}
$$

Proof. An immediate computation yields

$$
\frac{d}{d t} \Phi(t)=-\frac{1}{\kappa} \int_{0}^{\infty} \psi(s)\left\langle u(t), \partial_{t} \eta^{t}(s)\right\rangle d s-\frac{1}{\kappa} \int_{0}^{\infty} \psi(s)\left\langle\partial_{t} u(t), \eta^{t}(s)\right\rangle d s .
$$


Arguing exactly like in [18, Lemma 4.1], it is possible to prove the following control:

$$
\begin{aligned}
& -\frac{1}{\kappa} \int_{0}^{\infty} \psi(s)\left\langle u(t), \partial_{t} \eta^{t}(s)\right\rangle d s \\
& \leq-(1-\nu)\|u(t)\|^{2}-c_{2}\left(\int_{0}^{\infty} \mu^{\prime}(s)\left\|\eta^{t}(s)\right\|_{V}^{2} d s-\mathbb{J}\left[\eta^{t}\right]\right) .
\end{aligned}
$$

Concerning the second summand on the right-hand side of (6.6), we have

$$
\begin{aligned}
& -\frac{1}{\kappa} \int_{0}^{\infty} \psi(s)\left\langle\partial_{t} u(t), \eta^{t}(s)\right\rangle d s \\
& =\frac{1}{\kappa} \int_{0}^{\infty} \psi(s)\left(\int_{0}^{\infty} \mu(\zeta)\left\langle\eta^{t}(s), \eta^{t}(\zeta)\right\rangle_{V} d \zeta\right) d s \\
& \leq \frac{1}{\kappa}\left(\int_{0}^{\infty} \mu(s)\left\|\eta^{t}(s)\right\|_{V} d s\right)^{2} \\
& \leq \frac{1}{\kappa}\left(\int_{0}^{\infty}[\mu(s)]^{\frac{p-1}{p}} d s\right)\left(\int_{0}^{\infty}[\mu(s)]^{\frac{p+1}{p}}\left\|\eta^{t}(s)\right\|_{V}^{2} d s\right) \\
& \leq c_{1} \int_{0}^{\infty}[\mu(s)]^{\frac{p+1}{p}}\left\|\eta^{t}(s)\right\|_{V}^{2} d s,
\end{aligned}
$$

having used the fact that $(p-1) / p>1 / p$. The thesis is then achieved by collecting both of the above inequalities.

Lemma 6.5. Let $\beta=0$. For $M>0$ large enough, the functional defined by

$$
\mathcal{B}(t)=M \mathcal{E}(t)+\Phi(t)
$$

fulfills the inequalities

$$
K^{\prime} \mathcal{E}(t) \leq \mathcal{B}(t) \leq K \mathcal{E}(t)
$$

and

$$
\frac{d}{d t} \mathcal{B}(t)+C_{1}\|u(t)\|^{2}+C_{2} \int_{0}^{\infty}[\mu(s)]^{\frac{p+1}{p}}\left\|\eta^{t}(s)\right\|_{V}^{2} d s \leq 0
$$

for some positive constants $K, K^{\prime}, C_{1}$ and $C_{2}$.

Proof. Inequalities (6.7) follow immediately by the definition of $\Phi(t)$. In order to prove (6.8), we collect the energy identity (2.3) and (6.5), and by means of assumption (6.3), 
we have (for $M$ large enough)

$$
\begin{aligned}
\frac{d}{d t} \mathcal{B}(t) \leq & -(1-\nu)\|u(t)\|^{2}+c_{1} \int_{0}^{\infty}[\mu(s)]^{\frac{p+1}{p}}\left\|\eta^{t}(s)\right\|_{V}^{2} d s \\
& +\left(M-c_{2}\right)\left(\int_{0}^{\infty} \mu^{\prime}(s)\left\|\eta^{t}(s)\right\|_{V}^{2} d s-\mathbb{J}\left[\eta^{t}\right]\right) \\
\leq & -(1-\nu)\|u(t)\|^{2}+c_{1} \int_{0}^{\infty}[\mu(s)]^{\frac{p+1}{p}}\left\|\eta^{t}(s)\right\|_{V}^{2} d s \\
& -\delta\left(M-c_{2}\right) \int_{0}^{\infty}[\mu(s)]^{\frac{p+1}{p}}\left\|\eta^{t}(s)\right\|_{V}^{2} d s-\left(M-c_{2}\right) \mathbb{J}\left[\eta^{t}\right] .
\end{aligned}
$$

Inequality (6.8) then follows, provided that we choose $M>c_{2}+c_{1} / \delta$.

REmark 6.6. In the case $\beta>0$, simply set $\mathcal{B}(t)=\mathcal{E}(t)$. Then, by virtue of (6.3) along with the Poincaré inequality, from the energy identity (2.3) we easily get

$$
\frac{d}{d t} \mathcal{B}(t) \leq-\delta \int_{0}^{\infty}[\mu(s)]^{\frac{p+1}{p}}\left\|\eta^{t}(s)\right\|_{V}^{2} d s-2 \beta \lambda\|u(t)\|^{2},
$$

so that (6.7) and (6.8) follow.

Proof of Theorem 6.2, Let $\sigma \in(0,1)$. The Hölder inequality entails

$$
\begin{aligned}
\left\|\eta^{t}\right\|_{\mathcal{M}}^{2} & =\int_{0}^{\infty}[\mu(s)]^{\sigma}[\mu(s)]^{1-\sigma}\left\|\eta^{t}(s)\right\|_{V}^{2} d s \\
& \leq\left(\int_{0}^{\infty}[\mu(s)]^{\frac{\sigma(p+1)}{1+\sigma p}}\left\|\eta^{t}(s)\right\|_{V}^{2} d s\right)^{\frac{1+\sigma p}{p+1}}\left(\int_{0}^{\infty}[\mu(s)]^{\frac{p+1}{p}}\left\|\eta^{t}(s)\right\|_{V}^{2} d s\right)^{\frac{p-\sigma p}{p+1}} .
\end{aligned}
$$

Using once more the boundedness of $\left\|\eta^{t}(s)\right\|_{V}$, which holds after Remark 6.3, under the restriction

$$
\sigma(p+1) /(1+\sigma p)>1 / p
$$

we infer

$$
\left\|\eta^{t}\right\|_{\mathcal{M}}^{2 q} \leq C_{q}^{q} \int_{0}^{\infty}[\mu(s)]^{\frac{p+1}{p}}\left\|\eta^{t}(s)\right\|_{V}^{2} d s
$$

for any fixed $q>p /(p-1)$. The constant $C_{q}$ plays the same role as in Section 5 it depends increasingly on $\mathcal{E}(0)$ and singularly on $q$. So let us fix $q \in(p /(p-1), 1)$; then, by means of (6.7) and (6.9), and since $q>1$, we are led to

$$
\begin{aligned}
\mathcal{B}(t)^{q} & \leq K^{q} \mathcal{E}(t)^{q} \leq \mathcal{Q}\left[\|u(t)\|^{2 q}+\left\|\eta^{t}\right\|_{\mathcal{M}}^{2 q}\right] \\
& \leq \mathcal{Q}\left[\|u(t)\|^{2}+\int_{0}^{\infty}[\mu(s)]^{\frac{p+1}{p}}\left\|\eta^{t}(s)\right\|_{V}^{2} d s\right] .
\end{aligned}
$$

Therefore, from (6.8) we obtain

$$
\frac{d}{d t} \mathcal{B}(t)+\frac{1}{\mathcal{Q}} \mathcal{B}(t)^{q} \leq 0
$$

which, together with (6.7), yields

$$
\mathcal{E}(t) \leq \mathcal{Q}(1+t)^{-r} .
$$


From the limitation $q>p /(p-1)$, we immediately infer $r<p-1$. Again, we have a singular dependence of $\mathcal{Q}$ on $r$.

Acknowledgments. The authors wish to thank Professor Vittorino Pata for having raised the problem and for many fruitful discussions.

\section{REFERENCES}

[1] F. Ammar-Khodja, A. Benabdallah, J. E. Muñoz Rivera, R. Racke, Energy decay for Timoshenko systems of memory type, J. Differential Equations 194, 82-115 (2003). MR2001030 (2004f:74032)

[2] C. Cattaneo, Sulla conduzione del calore, Atti Sem. Mat. Fis. Univ. Modena 3, 83-101 (1948). MR0032898 (11:362d)

[3] V. V. Chepyzhov, E. Mainini, V. Pata, Stability of abstract linear semigroups arising from heat conduction with memory, Asymptot. Anal. 50, 269-291 (2006). MR2294601 (2007k:35284)

[4] V. V. Chepyzhov, V. Pata, Some remarks on stability of semigroups arising from linear viscoelasticity, Asymptot. Anal. 46, 251-273 (2006). MR2215885 (2007c:47053)

[5] B. D. Coleman, M. E. Gurtin, Equipresence and constitutive equations for rigid heat conductors, Z. Angew. Math. Phys. 18, 199-208 (1967). MR0214334(35:5185)

[6] M. Conti, S. Gatti, V. Pata, Uniform decay properties of linear Volterra integro-differential equations, Math. Models Methods Appl. Sci. 18, 21-45 (2008). MR.2378082

[7] C. M. Dafermos, Asymptotic stability in viscoelasticity, Arch. Rational Mech. Anal. 37, 297-308 (1970). MR0281400 (43:7117)

[8] M. Fabrizio, G. Gentili, D.W. Reynolds, On rigid linear heat condution with memory, Int. J. Eng. Sci. 36, 765-782 (1998). MR1629806 (99i:80006)

[9] M. Fabrizio, S. Polidoro, Asymptotic decay for some differential systems with fading memory, Appl. Anal. 81, 1245-1264 (2002). MR1956060(2004a:45015)

[10] C. Giorgi, G. Gentili, Thermodynamic properties and stability for the heat flux equation with linear memory, Quart. Appl. Math. 51, 343-362 (1993). MR1218373 (94j:80004)

[11] C. Giorgi, M.G. Naso, V. Pata, Exponential stability in linear heat conduction with memory: A semigroup approach, Comm. Appl. Anal. 5, 2001 (121-134). MR1844676 (2002e:35233)

[12] M. Grasselli, V. Pata, Uniform attractors of nonautonomous systems with memory, in "Evolution Equations, Semigroups and Functional Analysis" (A. Lorenzi and B. Ruf, Eds.), pp. 155-178, Progr. Nonlinear Differential Equations Appl. no. 50, Birkhäuser, Boston (2002). MR.1944162 (2003j:37135)

[13] M. E. Gurtin, A. C. Pipkin, A general theory of heat conduction with finite wave speeds, Arch. Rational Mech. Anal. 31, 113-126 (1968). MR.1553521

[14] R. K. Miller, An integro-differential equation for rigid heat conductors with memory, J. Math. Anal. Appl. 66, 313-332 (1978). MR515894 (80g:45015)

[15] J. E. Muñoz Rivera, E. Cabanillas Lapa, Decay rates of solutions of an anisotropic inhomogeneous $n$-dimensional viscoelastic equation with polynomially decaying kernels, Commun. Math. Phys. 177, 583-602 (1996). MR1385077 (97e:73034)

[16] J. E. Muñoz Rivera, M.G. Naso, E.Vuk, Asymptotic behaviour of the energy for electromagnetic systems with memory, Math. Methods Appl. Sci. 27, 819-841 (2004). MR2055321 (2005a:35268)

[17] J. E. Muñoz Rivera, R. Racke, Magneto-thermo-elasticity - large-time behavior for linear systems, Adv. Differential Equations 6, 359-384 (2001). MR1799490(2001j:74037)

[18] V. Pata, Exponential stability in linear viscoelasticity, Quart. Appl. Math. 64, 499-513 (2006). MR2259051 (2007h:35211)

[19] V. Pata, A. Zucchi, Attractors for a damped hyperbolic equation with linear memory, Adv. Math. Sci. Appl. 11, 505-529 (2001). MR1907454 (2003f:35027)

[20] A. Pazy, Semigroup of linear operators and application to partial differential equations, SpringerVerlag, New York, 1983. MR710486 (85g:47061) 